Table 1. Pregnancy outcomes

\begin{tabular}{lccc}
\hline Pregnancy, $\mathrm{n}$ & SLE, $\mathrm{n}=16$ & Control, $\mathrm{n}=15$ & $p$ \\
\hline Birth & $9(56,3 \%)$ & $13(87 \%)$ & 0,00 \\
Abortion & $2(12,5 \%)$ & 0 & 0,00 \\
Unfavorable pregnancy outcome & $5(31,2 \%)$ & $2(13 \%)$ & 0,00 \\
Missed miscarriage & $4(25 \%)$ & 0 & 0,00 \\
Spontaneous abortion & $1(6 \%)$ & $2(13 \%)$ & ns \\
\hline
\end{tabular}

Analysis of gynecological history indicate that episodes of menstrual disorders were significantly more often reported in SLE pts $(50 \%$ vs $20 \%$ in controls, $p=0.001$ ), similarly, gynecological diseases were also documented in $50 \%$ of SLE pts (chronic salpyngo-oophoritis, colpitis, endometriosis and uterine endometrioma, subserous uterine myoma, cervical dysplasia, cervical erosion), meanwhile low AMH was found only in 4 SLE pts; there was only 1 subject with gynecological condition - teratoma of the ovaries - in the control group (favorable outcome - surgical removal, preserved fertility and two births after surgery). Conclusion: The incidence of unfavorable pregnancy outcomes is significantly higher in patients with SLE compared to the control group of healthy women. Disclosure of Interests: None declared DOI: 10.1136/annrheumdis-2021-eular.3424

\section{AB0087 AUTOANTIBODIES DIRECTED AGAINST HOMOCYSTEINYLATED ALPHA 1 ANTITRYPSIN AS A POTENTIAL NEW BIOMARKER FOR ARTHRITIS IN PATIENTS AFFECTED BY SYSTEMIC LUPUS ERYTHEMATOSUS}

F. Natalucci ${ }^{1,1}$, F. Ceccarelli ${ }^{1}$, T. Colasanti ${ }^{1}$, G. Olivieri ${ }^{1}$, A. I. Celia ${ }^{1}$, C. Barbati ${ }^{1}$ M. Speziali ${ }^{1}$, F. Ucci ${ }^{1}$, C. Pirone ${ }^{1}$, C. Ciancarella ${ }^{1}$, F. R. Spinelli ${ }^{1}$, C. Alessandri ${ }^{1}$, F. Conti ${ }^{1}{ }^{1}$ Policlinico Umberto I, Sapienza University of Rome, Department of Clinical, Internal Medicine, Anesthesiology and Cardiovascular Sciences, Rome, Italy

Background: Joint involvement represents one of the most frequent features in patients affected by Systemic Lupus Erythematosus (SLE). This manifestation is characterized by a great heterogeneity in phenotype and severity: the application of more sensitive imaging techniques identified an erosive damage in about $25 \%$ of patients (1). This damage has been associated with autoantibodies, such as anti-citrullinated (ACPA) and anti-carbamylated proteins (antiCarP), previously identified in patients Rheumatoid Arthritis (RA) patients. Recently, homocysteinylated alpha 1 antitrypsin (Hcy-1A1AT) has been identified as a new antigenic target of autoantibodies in seronegative RA patients: in detail, anti-homocysteinylated alpha 1 antitrypsin (anti - HATA) antibodies have been identified in $75.7 \%$ of patients (2). Objectives: In the present study, we aimed at determining the prevalence of anti - HATA in a cohort of SLE patients.

Methods: We evaluated patients affected by SLE according to the 1997 ACR criteria. Demographic, clinical, and laboratory data were collected in a standardized computerized electronically filled form. Each subject underwent peripheral blood sample collection. Hcy-A1AT was obtained by in vitro modification of native A1AT and used as antigens by ELISA to test the presence of anti-HATA in sera obtained from enrolled subjects. Finally, we investigated the presence of ACPA and Rheumatoid Factor (RF) commercial ELISA kits and of anti-CarP (homemade ELISA) by a home-made ELISA in SLE patients' sera. As control, we enrolled 40 patients affected by Osteoarthritis (OA) and 41 healthy subjects (HS). Results: The present analysis included 88 SLE patients (M/F 6/82 median age 47 years (IQR 17), median disease duration 156 months (IQR 180). Joint involvement was observed in 75 SLE patients (85.2\%): in detail, 65 patients referred arthritis and the remaining 10 inflammatory arthralgias. We identified the presence of anti-HATA IgG in 38 SLE patients (43.2\%). This prevalence was significantly higher in comparison with OA and HS subjects [15.0\% $(p<0.001)$ and $0 \%(p<0.0001)$, respectively; Figure $1 \mathrm{~A}]$. Focusing on the SLE cohort, no differences were observed between patients with and without joint involvement in anti-HATA IgG prevalence $(41.3 \%$ versus $34.7 \%$, respectively; $p=0.34)$. However considering SLE patients according to the presence of arthralgia and arthritis, the prevalence of anti-HATA was significantly higher in patients with arthritis in comparison with those patients with arthralgias $(46.1 \%$ versus $11.1 \%$, $\mathrm{p}=0.02$; figure $1 \mathrm{~B}$ ). Finally, no significant association between anti-HATA and the other tested autoantibodies (RF, ACPA, anti-CarP) was found.
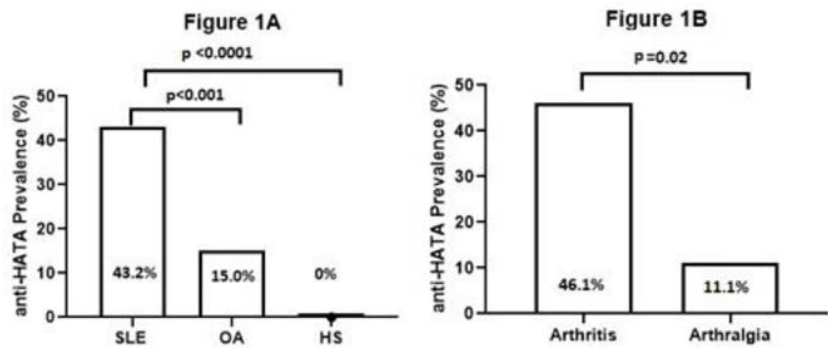

Conclusion: We evaluated the prevalence of anti-HATA in a cohort of SLE patients. The prevalence of these autoantibodies was significantly higher in SLE patients than in OA patients and in HS. The association with arthritis suggests a possible role for anti-HATA as biomarkers of SLE-related joint involvement.

\title{
REFERENCES:
}

[1] Ceccarelli F. Perricone C. Cipriano E. et al. Joint involvement in systemic lupus erythematosus: From pathogenesis to clinical assessment. Seminar in Arthritis and Rheumatism, 47(1), $53-64$

[2] Colasanti T. Sabatinelli D. Mancone et al. Homocysteinylated alpha 1 antitrypsin as an antigenic target of autoantibodies in seronegative rheumatoid arthritis patients. Journal of Autoimmunity 2020 Sep;113:102470.

Disclosure of Interests: None declared

DOI: 10.1136/annrheumdis-2021-eular.3688

\section{$\mathrm{AB} 0088$ \\ QUALITY OF LIFE IN PATIENTS WITH ANTIPHOSPHOLIPID ANTIBODIES DIFFERS ACCORDING TO ANTIPHOSPHOLIPID SYNDROME DAMAGE INDEX (DIAPS)}

S. G. Foddai ${ }^{1,2}$, M. Radin ${ }^{1,2}$, A. Barinotti ${ }^{1,2}$, I. Cecchi $^{1,2}$, E. Rubini ${ }^{1,2}$,

S. Sciascia ${ }^{1,2}$, D. Roccatello ${ }^{1,2}$. ${ }^{1}$ Turin, Università di Torino, Turin, Italy; ${ }^{2}$ Turin, Center of Research of Immunopathology and Rare Diseases - Coordinating Center of Piemonte and Valle d'Aosta Network for Rare Diseases, Department of Clinical and Biological Sciences, Turin, Italy

Background: Antiphospholipid syndrome (APS) is a rare autoimmune disorder that mainly affects young individuals. Due to the potentially devasting effects of some of its clinical manifestations, such as ischemic stroke and thrombotic microangiopathy, APS can have a deep negative impact on the quality of life (QoL) of the affected patients due to damage accrual (2).

To capture the full consequences of the disease, including both thrombotic and extra-criteria APS specific features, the antiphospholipid syndrome (APS) damage index (DIAPS), a 37-item score, was conceived.

Objectives: The aim of this study was to validate the DIAPS in a cohort of antiphospholipid antibodies (aPL) positive subjects, comparing it to the risk stratification score for the development of clinical manifestations of APS (Global APS Score -GAPSS) and QoL questionnaires filled out by APS patients.

Methods: This retrospective study included a total of 84 consecutive persistent aPL positive patients who attended the San Giovanni Bosco Hospital (Turin, Italy) Patients were then divided based on their diagnosis in 3 groups: primary APS (PAPS), secondary APS (SAPS) and aPL positive patients without clinical manifestations of APS, according to Miyakis criteria (1). Demographic, clinical and laboratory characteristics, were retrieved from electronic medical records and are summarized in Table 1. DIAPS was calculated as previously reported by adding together all points corresponding to the clinical manifestations of the patients (3). Results: A total of consecutive 84 patients were enrolled in the study: 39 primary APS patients (PAPS), 28 secondary APS patients (SAPS) and 17 aPL positive patients without APS-related clinical manifestations. APS patients had significantly higher levels of DIAPS when compared to aPL asymptomatic patients (mean DIAPS $2.6 \pm 1.8$ vs. $1.5 \pm 1.9$, respectively; $p<0.05$ ). Moreover, SAPS patients had significantly higher levels of DIAPS when compared to PAPS patients (mean DIAPS $3.1 \pm 1.9$ vs. $2.2 \pm 1.7$, respectively; $p<0.05$ ). When comparing GAPSS and DIAPS levels in all the patients, we found a significantly positive correlation between these two scores (mean GAPSS $11.9 \pm 5.2$ and mean DIAPS $2.4 \pm 1.9$; Pearson $0.241 ; \mathrm{p}<0.05$ ). Finally, when applying the SF-12 score to our cohort, the mean physical component score and mental component score were lower than the average population $(39.3 \pm 11$.3 and $42.3 \pm 8$, respectively) and we observed a negative correlation trend between DIAPS and both PCS and MCS (Pearson -0.133 and -0.183).

Table 1. Clinical and laboratory characteristics of the patients

\begin{tabular}{lll}
\hline PAPS & SAPS & aPL asymptomatic \\
$(n=39)$ & $(n=28)$ & $(n=17)$
\end{tabular}

Anagraphic

Mean age (S.D.)

Sex (females), $n$

Secondary diagnosis, $\mathrm{n}$

Clinical manifestations of APS

Thrombosis, $n$

Arterial thrombosis, $n$

Venous thrombosis, $n$

Pregnancy morbidity, $\mathrm{n}$

aPL profile at diagnosis

LA, $n$

aCL (IgG/M), n

Anti-p2GPI (IgG/M),

aPS/PT (IgG/M), n

$(n=28)$ $(\mathrm{n}=17)$ $50.6 \pm 13.7$
$29(74.4 \%)$ N/A

$47.4 \pm 12$ 29 (74.4\%) SLE $8(45.1 \%)$ SSJ 1 (3.6\%) UCTD $1(3.6 \%)$ MCTD 2 (7.1\%)

$30(76.9 \%) \quad 26(92.9 \%) \quad N / A$ $20(51.3 \%) \quad 14(50 \%)$ $17(43.6 \%) \quad 15(53.6 \%)$

$11(28.2 \%) \quad 4(14.3 \%)$

$31(79.5 \%) \quad 23(82.1 \%)$

$22(56.4 \%) \quad 18(64.3 \%)$

$18(46.2 . \%) \quad 16(57.1 \%)$

$23(59 \%) \quad 21(75 \%)$
$\mathrm{N} / \mathrm{A}$

N/A

$12(70.6 \%)$

$10(58.9 \%)$

$3(17.6 \%)$

$8(47.1 \%)$ 
Conclusion: The DIAPS might be a valid score system for the assessment of APS-related damage and QoL.

REFERENCES:

[1] Miyakis S, Lockshin MD, et al. International consensus statement on an update of the classification criteria for definite antiphospholipid syndrome (APS). J Thromb Haemost 2006;4:295-306.

[2] Grika EP, Ziakas PD, et al. Morbidity, mortality, and organ damage in patients with antiphospholipid syndrome. J Rheumatol 2012;39:516-523.

[3] Amigo MC, Goycochea-Robles M V. et al. Development and initial validation of a damage index (DIAPS) in patients with thrombotic antiphospholipid syndrome (APS). Lupus 2015;24:927-934.

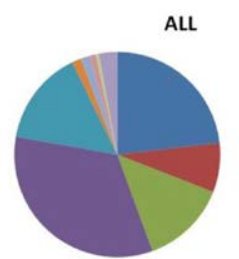

aPL
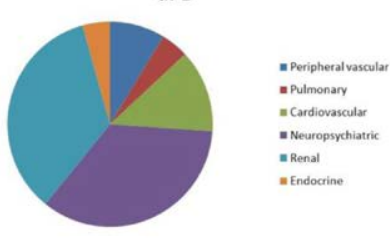

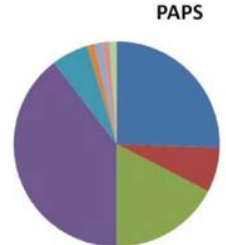

SAPS
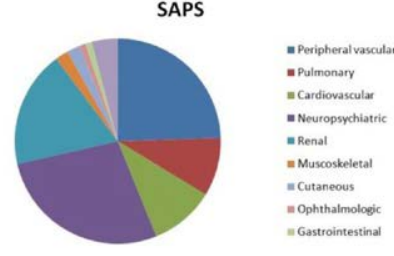

Figure 1. Relative involvement of the organ domains included in the DIAPS according to diagnosis

Disclosure of Interests: None declared

DOI: 10.1136/annrheumdis-2021-eular.4295

\section{Systemic sclerosis, myositis and related syndromes - aetiology, pathogenesis and animal models.}

\section{AB0089 SILDENAFIL COUNTERACTS THE ACTIVATION OF CXCR3/ CXCL10,-11 AXIS IN SCLERODERMA FIBROBLASTS EXPOSEDTO REACTIVE OXYGEN SPECIES}

C. Antinozzi ${ }^{1}$, I. Dimauro ${ }^{1}$, P. Sgrò ${ }^{1}$, D. Caporossi ${ }^{1}$, F. Del Galdo ${ }^{2,3}$, L. DI Luigi ${ }^{1}$. ${ }^{1}$ University of Rome Foro Italico, Movement, Human and Health Sciences, Rome, Italy; ${ }^{2}$ University of Leeds, Institute of Rheumatic and Musculoskeletal Diseases, Leeds, United Kingdom; ${ }^{3}$ NIHR Biomedical Research Centre, University of Leeds, Leeds, United Kingdom

Background: Oxidative stress associated with vascular damage represents one the major contributor in the pathogenesis of systemic sclerosis (SSc) [1]. Indeed, different studies demonstrated that excessive free radicals production can contribute to the activation of fibrotic process in the skin and visceral organs [1]. CXCL10 and CXCL11, together with their receptor CXCR3, are involved in vascular damage and in fibrosis [2]. Furthermore, these chemokines have been proposed as biomarkers of vascular damage progression and severe SSc prognosis [3].

Emerging evidences highlight the beneficial effects of the phosphodiesterase type 5 (PDE5) inhibitor, sildenafil, to protect different cell types from reactive oxygen species (ROS)-induced DNA damage, in vitro [3]. This effect has been linked to modulation of CXCL10 concentration in different pathological conditions $[4,5]$. Objectives: Here we set out to investigate the effects of sildenafil, in modulating the CXCR3/CXCL10, -11 inflammatory axis in dermal fibroblasts exposed to oxidative stress, in vitro.

Methods: Human dermal fibroblasts isolated by SSc skin biopsies were treated for $24 \mathrm{~h}$ with $100 \mu \mathrm{M}$ of hydrogen peroxide $\left(\mathrm{H}_{2} \mathrm{O}_{2}\right)$, in the presence or not of sildenafil $(1 \mu \mathrm{M})$. Dermal fibrobalsts from healthy skin were used as controls. CXCL10 and CXCL11 were evaluated in cell medium by luminex technology assay; expression of chemokine receptor (CXCR)3 and peroxisome proliferator-activated receptor (PPAR $\gamma$ ) (a regulator of CXCL10,-11 mRNA) was evaluated by western blot assay. Results: As showed in figure 1, SSc fibroblasts (grey bar) showed similar basal levels of CXCL10 (A) and CXCL11 (B) to healthy controls (black bar). $\mathrm{H}_{2} \mathrm{O}_{2}$ induced a significant increase of both chemokines only in SSc fibroblasts (by 4.6 fold for CXCL10 and by 4.2 fold for CXCL11) $\left({ }^{*} P<0.05\right.$ and ${ }^{* \star} P<0.01$ vs. $C$; ${ }^{\#} \mathrm{P}<0.05$ vs. healthy controls). Sildenal pre-incubation reduced by approximatively $50 \%$ the effects of $\mathrm{H}_{2} \mathrm{O}_{2}$ on chemokines release (Figure $1 \mathrm{~A}$ and $\left.\mathrm{B}\right)\left({ }^{\S} \mathrm{P}<0.05\right.$ vs. $\mathrm{H}_{2} \mathrm{O}_{2}$ ), and reduced the expression of CXCR3 and PPAR $\gamma$ induced by hydrogen peroxyde exposure (data not shown).
Conclusion: In vitro study on dermal fibroblasts support clinical studies to determine the efficacy of sildenafil in the preventing tissue damage and fibrosis in $\mathrm{SSc}$, by reducing the pro-inflammatory activation induced by oxidative stress. REFERENCES:

[1] Di Luigi L, Sgrò P, Duranti G, Sabatini S, Caporossi D, Del Galdo F, Dimauro I, Antinozzi C. Sildenafil Reduces Expression and Release of IL-6 and IL-8 Induced by Reactive Oxygen Species in Systemic Sclerosis Fibroblasts. Int J Mol Sci. 2020 Apr 30;21(9):3161. doi: 10.3390/ijms21093161. PMID: 32365773; PMCID: PMC7246497.

[2] Koper OM, Kamińska J, Sawicki K, Kemona H. CXCL9, CXCL10, CXCL11, and their receptor (CXCR3) in neuroinflammation and neurodegeneration. Adv Clin Exp Med. 2018 Jun;27(6):849-856. doi: 10.17219/acem/68846. PMID: 29893515.

[3] Crescioli C, Corinaldesi C, Riccieri V, Raparelli V, Vasile M, Del Galdo F, Valesini G, Lenzi A, Basili S, Antinozzi C. Association of circulating CXCL10 and CXCL11 with systemic sclerosis. Ann Rheum Dis. 2018 Dec;77(12):18451846. doi: 10.1136/annrheumdis-2018-213257. Epub 2018 May 14. PMID: 29760155; PMCID: PMC6241615.

[4] Giannattasio S, Corinaldesi C, Colletti M, Di Luigi L, Antinozzi C, Filardi T, Scolletta S, Basili S, Lenzi A, Morano S, Crescioli C. The phosphodiesterase 5 inhibitor sildenafil decreases the proinflammatory chemokine IL- 8 in diabetic cardiomyopathy: in vivo and in vitro evidence. J Endocrinol Invest. 2019 Jun;42(6):715-725. doi: 10.1007/s40618-018-0977-y. Epub 2018 Nov 10. PMID: 30415310; PMCID: PMC6531405.

[5] You N, Li J, Huang X, Wu K, Tang Y, Wang L, Li H, Mi N, Zheng L. COMMD7 activates $C X C L 10$ production by regulating NF- $\mathrm{KB}$ and the production of reactive oxygen species. Mol Med Rep. 2018 May;17(5):6784-6788. doi: 10.3892/mmr.2018.8706. Epub 2018 Mar 8. PMID: 29532873.
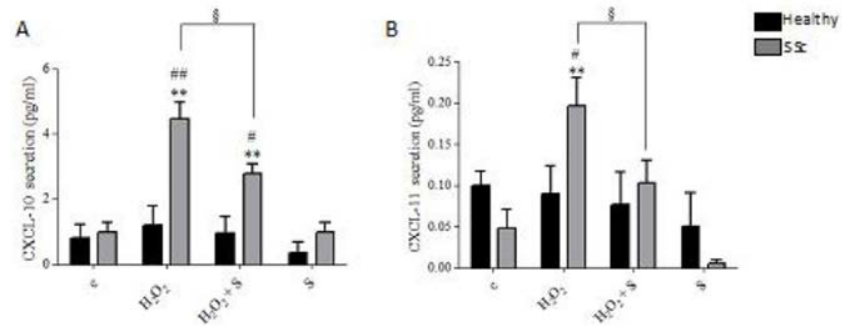

Disclosure of Interests: None declared

DOI: 10.1136/annrheumdis-2021-eular.459

\section{AB0090}

\section{THE BIOCHEMICAL BASIS OF ANIMAL MODEL OF} SYSTEMIC SCLEROSIS

B. Doskaliuk ${ }^{1}$, L. Zaiats ${ }^{1}$, R. Yatsyshyn ${ }^{2} .{ }^{1}$ Ivano-Frankivsk National Medical University, Pathophysiology, Ivano-Frankivsk, Ukraine; ${ }^{2}$ Ivano-Frankivsk National Medical University, Internal Medicine \#1 Clinical Immunology and Allergology, Ivano-Frankivsk, Ukraine

Background: Systemic sclerosis (SSc) is a heterogeneous autoimmune pathology, which detailed pathogenetic mechanisms of initiation and progression still require further investigations. It could be achieved with implementation of reliable experimental modeling techniques.

Objectives: This study was aimed to determine the state of oxidant/antioxidant system, dysregulation of collagen synthesis in laboratory animals and evaluate effectiveness of the considered model of SSc induction.

Methods: Thirty adult Wistar rats $(220-240 \mathrm{~g})$ were included in the study. The experimental group consisted of 20 animals, which received 3 subcutaneous injections of $0.5 \mathrm{ml}$ of $5 \%$ sodium hypochlorite solution $(\mathrm{NaClO})$ with an active chlorine concentration of $190 \mathrm{~g} / \mathrm{dm}^{3}$ for 6 consecutive weeks. The control group consisted of 10 rats injected with isotonic solution according to the previously described scheme.

Results: The level of catalase in the experimental group was lower than in the control $9.69 \pm 0.67 \mathrm{~g} / \mathrm{I}$ and $11.1 \pm 0.87 \mathrm{~g} / \mathrm{I}$ respectively $(\mathrm{p}=0.002)$. Protein oxidative modification (POM) products were higher in the experimental group $(0.0722$ \pm 0.023 vs $0.0527 \pm 0.023, p=0.014)$. Malondialdehyde (MDA) was also higher in the experimental group compared to the control $(4.30 \pm 0.26$ and $3.85 \pm 0.23$, respectively, $p=0.010$ ). The concentration of oxyproline in the serum of laboratory animals subjected to repeated administration of $\mathrm{NaClO}$ was significantly higher compared to the control group $(34.03 \pm 5.01$ and $33.23 \pm 2.40, p=0.020)$. Conclusion: As a result of the use of $\mathrm{NaClO}$ for the reproduction of $\mathrm{SSc}$ in laboratory animals, there is a violation of oxidative-antioxidant homeostasis and increased fibrotic processes. These changes are comparable with those in SSc patients, which makes this modeling technique a promising tool for a detailed investigation of SSc pathogenesis.

Disclosure of Interests: None declared

DOI: 10.1136/annrheumdis-2021-eular.732 